\title{
UNIFORM SPARSE DOMINATION OF SINGULAR INTEGRALS VIA DYADIC SHIFTS
}

\author{
AMALIA CULIUC, FRANCESCO DI PLINIO, AND YUMENG OU
}

\begin{abstract}
Aвstract. Using the Calderón-Zygmund decomposition, we give a novel and simple proof that $L^{2}$ bounded dyadic shifts admit a domination by positive sparse forms with linear growth in the complexity of the shift. Our estimate, coupled with Hytönen's dyadic representation theorem, upgrades to a positive sparse domination of the class $\mathcal{U}$ of singular integrals satisfying the assumptions of the classical $T(1)$-theorem of David and Journé. Furthermore, our proof extends rather easily to the $\mathbb{R}^{n}$-valued case, yielding as a corollary the operator norm bound on the matrix weighted space $L^{2}\left(W ; \mathbb{R}^{n}\right)$
\end{abstract}

$$
\left\|T \otimes \operatorname{Id}_{\mathbb{R}^{n}}\right\|_{L^{2}\left(W ; \mathbb{R}^{n}\right) \rightarrow L^{2}\left(W ; \mathbb{R}^{n}\right)} \lesssim[W]_{A_{2}}^{\frac{3}{2}}
$$

uniformly over $T \in \mathcal{U}$, which is the currently best known dependence.

\section{Main Results and context}

Set in motion by the seminal article of Andrei Lerner [18], the pointwise control of singular integral operators by positive sparse averages of the input functions has proved to be a remarkably effective strategy towards sharp weighted norm inequalities, within and beyond Calderón-Zygmund theory.

In this note, we set forth a novel and simple approach to positive sparse domination of singular integral operators, at the core of which lies the classical Calderón-Zygmund decomposition. Our approach has the advantage of extending rather effortlessly to the case of singular integrals acting on $\mathbb{R}^{n}$-valued functions, thus yielding matrix weighted norm inequalities with quantified dependence on the matrix weight characteristic. We provide additional context after the statement of our main results.

1.1. Main results. Our first domination result, Theorem 1, involves dyadic shifts, which are the fundamental discrete model for Calderón-Zygmund operators. We send to Hytönen [11] and references therein for more precise versions of this statement, and proceed to the formal definition. Let $\mathcal{D}$ be a dyadic lattice on $\mathbb{R}^{d}$, and $m_{1}, m_{2}$ be two nonnegative integers. A bilinear form

$$
\mathbb{S}^{\varrho}\left(f_{1}, f_{2}\right)=\sum_{Q \in \mathcal{D}} S_{Q}\left(f_{1}, f_{2}\right), \quad S_{Q}\left(f_{1}, f_{2}\right):=\int_{Q \times Q} s_{Q}\left(x_{1}, x_{2}\right) f_{1}\left(x_{1}\right) f_{2}\left(x_{2}\right) \mathrm{d} x_{1} \mathrm{~d} x_{2}
$$

2010 Mathematics Subject Classification. Primary: 42B20. Secondary: 42B25.

Key words and phrases. Positive sparse operators, T(1) theorems, weighted norm inequalities, matrix weights.

F. Di Plinio was partially supported by the National Science Foundation under the grants NSF-DMS-1500449 and NSF-DMS-1650810. 
defined for $f_{j} \in L_{\text {loc }}^{1}\left(\mathbb{R}^{d}\right), j=1,2$ is termed a dyadic shift of complexity $\varrho=\max \left\{1, m_{1}, m_{2}\right\}$ constructed on $\mathcal{D}$ if the following assumptions hold:

A1. $s_{Q}: Q \times Q \rightarrow \mathbb{C}$ is zero for all but finitely many $Q \in \mathcal{D}$ and $\left\|s_{Q}\right\|_{\infty} \leq|Q|^{-1}$.

A2. there holds

$$
\sup _{\mathcal{Q} \subset \mathcal{D}}\left|\mathbb{S}_{\mathcal{Q}}^{\varrho}\left(f_{1}, f_{2}\right)\right| \leq\left\|f_{1}\right\|_{2}\left\|f_{2}\right\|_{2}
$$

where the subshifts $\mathbb{S}_{\mathcal{Q}}^{\varrho}$ are defined by

$$
\mathbb{S}_{\mathcal{Q}}^{\varrho}\left(f_{1}, f_{2}\right):=\sum_{Q \in \mathcal{Q}} S_{Q}\left(f_{1}, f_{2}\right)
$$

A3. if $R \in \mathcal{D}, R \subset Q$ and $\ell(R)<2^{-m_{1}} \ell(Q)$ then $s_{Q}\left(\cdot, x_{2}\right)$ is constant on $R$ for all $x_{2} \in Q$, and symmetric assumption with the roles of $x_{1}, x_{2}$ interchanged.

We now introduce the ingredients of a positive sparse form. For a cube $Q \subset \mathbb{R}^{d}$, we write

$$
\langle f\rangle_{Q}:=\frac{1}{|Q|} \int_{Q}|f| \mathrm{d} x \text {. }
$$

We say that a collection $\mathcal{S}$ of cubes of $\mathbb{R}^{d}$ is $\eta$-sparse if for each $Q \in \mathcal{S}$ there exists $E_{Q} \subset Q$ with $\left|E_{Q}\right| \geq \eta|Q|$ and such that the sets $\left\{E_{Q}: Q \in \mathcal{S}\right\}$ are pairwise disjoint. The precise value of $\eta<1$ will be of no interest for us in what follows.

Theorem 1. There exists an absolute constant $C$ such that the following holds. For every $f_{1}, f_{2} \in L^{1}\left(\mathbb{R}^{d}\right)$ with compact support and every dyadic lattice $\mathcal{D}$ there exists a sparse collection $\mathcal{S}_{\mathcal{D}}$ such that for all $\varrho \geq 1$

$$
\sup _{\mathbb{S} \varrho}\left|\mathbb{S}^{\varrho}\left(f_{1}, f_{2}\right)\right| \leq C \varrho \sum_{Q \in \mathcal{S}_{\mathcal{D}}}|Q|\left\langle f_{1}\right\rangle_{Q}\left\langle f_{2}\right\rangle_{Q}
$$

the supremum being taken over all dyadic shifts $\mathbb{S}$ e of complexity $\varrho$ constructed on $\mathcal{D}$.

The proof is given in Section 2. We may upgrade the sparse domination of Theorem 1 to a domination of singular integrals satisfying smoothness assumptions on the kernel and David-Journé type testing conditions, by using the well-known representation principle of Hytönen [11], built upon previous work of Nazarov, Treil and Volberg [20].

Let $\mathcal{U}$ be the family of singular integral operators, acting on a dense subspace $\mathcal{W}$ of $L^{2}\left(\mathbb{R}^{d}\right)$ containing, say, bounded functions with compact support, and satisfying the following quantitative assumptions.

B1. Each $T \in \mathcal{U}$ has kernel representation

$$
T f(x)=\int_{\mathbb{R}^{d}} K(x, y) f(y) \mathrm{d} y, \quad x \notin \operatorname{supp} f
$$

with $K: \mathbb{R}^{d} \times \mathbb{R}^{d} \backslash\{(x, y): x=y\} \rightarrow \mathbb{C}$ satisfying the standard estimates

$$
\begin{aligned}
& |K(x, y)| \leq \frac{1}{|x-y|^{d}}, \\
& |K(x+h, y)-K(x, y)|+|K(x, y+h)-K(x, y)| \leq \frac{\omega\left(\frac{|h|}{|x-y|}\right)}{|x-y|^{d}} \quad \forall|h|<\frac{|x-y|}{2}
\end{aligned}
$$


where $\omega$ is the modulus of continuity $\omega(t)=t^{\alpha}, t \in(0,1]$, and $\alpha \in(0,1]$ is fixed.

B2. There holds

$$
\sup _{Q}\left|\left\langle T\left(\mathbf{1}_{Q}\right), \mathbf{1}_{Q}\right\rangle\right| \leq|Q|
$$

the supremum being taken over cubes $Q \subset \mathbb{R}^{d}$.

B3. With an appropriate definition of $T 1, T^{*} 1$, there holds

$$
\|T 1\|_{\mathrm{BMO}\left(\mathbb{R}^{d}\right)},\left\|T^{*} 1\right\|_{\mathrm{BMO}\left(\mathbb{R}^{d}\right)} \leq 1 .
$$

The following proposition is a restatement of the representation theorem from [11], in the more precise version provided in [10].

Proposition 1.1. [10, Theorem 3.3] Let $f_{1}, f_{2} \in \mathcal{W}$. There holds

$$
\sup _{T \in \mathcal{U}}\left|\left\langle T f_{1}, \overline{f_{2}}\right\rangle\right| \leq C \sup _{\varrho \geq 1} \sup _{\mathcal{D}, \mathbb{S} e} \varrho^{-1}\left|\mathbb{S}^{\varrho}\left(f_{1}, f_{2}\right)\right|
$$

with a constant $C>0$ depending on $d, \alpha$ only, the second supremum being taken over all dyadic lattices $\mathcal{D}$ of $\mathbb{R}^{d}$ and all dyadic shifts $\mathbb{S} e$ of complexity $\varrho$ constructed on $\mathcal{D}$.

Remark 1.2. In $[10,11]$, following ideas of $[20]$, the author constructs a family $\mathcal{D}_{\omega}$ of dyadic lattices parametrized by $\omega \in \Omega=\left(\{0,1\}^{d}\right)^{\mathbb{Z}}$. Then, Theorem 3.3 of [10] rewritten in our language yields that for each $T \in \mathcal{U}, f_{1}, f_{2} \in \mathcal{W}$, the equality

$$
\left\langle T f_{1}, \overline{f_{2}}\right\rangle=\mathbb{E}_{\omega} \sum_{\varrho=1}^{\infty} \tau(\varrho) \mathbb{S}_{\omega}^{\varrho}\left(f_{1}, f_{2}\right)
$$

holds with a suitable choice of $\mathbb{S} \underset{\omega}{\varrho}$, a dyadic shift of complexity $\varrho$ constructed on the dyadic lattice $\mathcal{D}_{\omega}$, and with a sequence $\{\tau(\varrho)\}$ satisfying

$$
|\tau(\varrho)| \leq C 2^{-\frac{\alpha}{2} \varrho}
$$

the expectation in (1.3) being taken over the natural probability measure on $\Omega$. The uniform estimate of Proposition 1.1 thus follows by dominating the right hand side of (1.3) by the right hand side of (1.2) times the series of $\varrho 2^{-\frac{\alpha}{2} \varrho}$, and summing the series.

Remark 1.3. We note that more refined versions of the representation formula of [10] may be employed to extend (1.2) to logarithmic-type moduli of continuity $\omega$; see for instance the very recent article [9]. However, these methods fall short of tackling the Dini-continuous case first settled in [14]. For this reason, and given that the main aim of this paper is to present a new sparse domination technique in the simplest possible setting, we choose to restrict our analysis to power-type moduli of continuity.

Coupling the domination Theorem 1 with Proposition 1.1 yields the following sparse domination theorem.

Theorem 2. There exists an absolute constant $C>0$ such that the following holds. For every $f_{1}, f_{2} \in \mathcal{W}$ and having compact support there exists a sparse collection $\mathcal{S}$ such that

$$
\sup _{T \in \mathcal{U}}\left|\left\langle T f_{1}, \overline{f_{2}}\right\rangle\right| \leq C \sum_{Q \in \mathcal{S}}|Q|\left\langle f_{1}\right\rangle_{Q}\left\langle f_{2}\right\rangle_{Q}
$$


Proof. Fix a pair of functions $f_{1}, f_{2} \in \mathcal{W}$ with compact support. A combination of Theorem 1 with Proposition 1.1 readily yields the inequality

$$
\sup _{T \in \mathcal{U}}\left|\left\langle T f_{1}, \overline{f_{2}}\right\rangle\right| \leq C \sup _{\mathcal{T}} \sum_{Q \in \mathcal{T}}|Q|\left\langle f_{1}\right\rangle_{Q}\left\langle f_{2}\right\rangle_{Q}
$$

the supremum being taken over all sparse collections $\mathcal{T}$. The proof of Theorem 2 is then finished by the observation that there exists a sparse collection $\mathcal{S}$ (depending only on $f_{1}, f_{2}$ ) such that

$$
\sup _{\mathcal{T}} \sum_{Q \in \mathcal{T}}|Q|\left\langle f_{1}\right\rangle_{Q}\left\langle f_{2}\right\rangle_{Q} \leq C \sum_{Q \in \mathcal{S}}|Q|\left\langle f_{1}\right\rangle_{Q}\left\langle f_{2}\right\rangle_{Q}
$$

The last claim follows via a simple stopping time argument based on the size of $\left\langle f_{1}\right\rangle_{Q}\left\langle f_{2}\right\rangle_{Q}$; we send to [15, Lemma 4.7] for the full proof.

Our proof of the dyadic shift domination Theorem 1 is based on a stopping time argument akin to the one employed by the authors in [7] to prove a uniform sparse domination theorem for the bilinear Hilbert transforms. At the heart of both lies a Calderón-Zygmund decomposition: classical, in Lemma 2.1 of this paper, around multiple frequencies in the outer $L^{p}$-embedding theorem [8] by two of us which is relied upon in [7].

Perhaps surprisingly, our approach extends effortlessly to singular integrals acting on functions taking values in a finite-dimensional Euclidean space, once a suitable vector valued version of the Calderón-Zygmund decomposition is introduced with Lemma 3.3. In Section 3, we adapt our proof of Theorem 1 to obtain uniform positive sparse domination of singular integrals in the class $\mathcal{U}$ : see Theorem 4. Besides its intrinsic interest, Theorem 4 also yields the currently best known quantitative matrix $A_{2}$ weighted estimates for the $\mathbb{R}^{n}$-valued extension of operators of the class $\mathcal{U}$ : see Corollary 5 . Our positive sparse forms in this setting involve the Minkowski product of convex sets generated by local averages of the input functions, a variation on a theme proposed by Nazarov, Petermichl, Treil and Volberg [22].

1.2. Context. We turn to a deeper description of the context and consequences of our approach. The pointwise control of a Calderón-Zygmund operator $T$ by $2^{d}$ sparse averaging operators depending on $T$ itself and on the input function $f$,

$$
|T f(x)| \leq C \sum_{1 \leq j \leq 2^{d}} \sum_{Q \in \mathcal{S}_{j}}\langle f\rangle_{Q} \mathbf{1}_{Q}(x)
$$

has been first achieved independently by Conde-Alonso and Rey [6] and Lerner and Nazarov [17], elaborating on Lerner's seminal paper [18]. A powerful approach to (1.4) forgoing the local mean oscillation estimate has been introduced by Lacey in [14] and subsequently streamlined by Lerner [16]. In contrast to all these previous works, the weak $(1,1)$ estimate for $T$ is not an a priori assumption of our Theorem 2. Rather, it is obtained as a consequence of the domination theorem from the standard assumptions of a $T(1)$ theorem. Furthermore, the sparse collection in Theorem 2 is explicitly constructed from level sets of the maximal function rather than the specific operator, and is thus the same for all operators in the class $\mathcal{U}$.

We also note that than the domination by sparse forms as in Theorem 2, while formally weaker, seems to be just as useful as the pointwise control (1.4). In fact, it is by dualizing 
(1.4) that the essential disjointness of $Q \in \mathcal{S}_{j}$ may be exploited. Hence, just as well as (1.4), Theorem 2 leads rather immediately to Hytönen's sharp weighted inequalities [11]

$$
\sup _{T \in \mathcal{U}}\|T\|_{L^{p}(w) \rightarrow L^{p}(w)} \leq C \frac{p}{p-1}[w]_{A_{p}}^{\max \left\{1, \frac{1}{p-1}\right\}} .
$$

See [19] for a self-contained argument deducing (1.5) from Theorem 2. A more general treatment is provided in [17, Section 16]. Comparing to the routes to the $A_{2}$-theorem outlined in the interesting survey [12], we believe that our approach provides an additional shortcut to a sharp weighted $T(1)$ theorem stemming directly from the representation theorem of [11]. It is likely that our proof strategy may be further applicable within the developing field of sparse domination in the nonhomogeneous and noncommutative setting: see [5] for a recent breakthrough result.

Concerning the vector-valued extension, quantified matrix $A_{2}$ estimates have appeared in the recent works $[1,2,4]$. A closely related result to Theorem 4, involving the Minkowski sum of convex body-valued sparse operators rather than bilinear forms, was announced by Nazarov, Petermichl, Treil and Volberg [22] before the present article was prepared. The details of their argument were unknown to us at the time of completion of the first version of this article, and were made public in the preprint [21], while our own article was being revised for publication.

Acknowledgements. The concept of domination by convex body averages originates from an idea of Fedor Nazarov. The authors want to thank Sergei Treil for introducing this idea to them during his seminar talk at Brown University in the Spring of 2016. The authors also extend their gratitude to Michael Lacey, Jill Pipher and Brett Wick for their comments on an early version of this manuscript, and to the anonymous referee for the valuable suggestions.

\section{Proof of Theorem 1}

Throughout this proof, we denote by $C$ a positive constant which is allowed to depend on the dimension $d$ only and whose value may vary from line to line without explicit mention.

2.1. Construction of the sparse collection $\mathcal{S}_{\mathcal{D}}\left(f_{1}, f_{2}\right)$. Below, we write $\mathcal{D}(Q):=\{R \in \mathcal{D}$ : $R \subset Q\}$. For $f_{1}, f_{2} \in L_{\text {loc }}^{1}\left(\mathbb{R}^{d}\right)$ and $Q \in \mathcal{D}$, we define $I \in \mathcal{I}_{Q}$ to be the maximal elements of $\mathcal{D}(Q)$ such that

$$
\left\langle f_{j}\right\rangle_{I}>2^{8}\left\langle f_{j}\right\rangle_{Q} \quad \text { for at least one } j=1,2 \text {. }
$$

Then

$$
\sum_{I \in \mathcal{I}_{Q}}|I| \leq 2^{-7}|Q|
$$

Now, if $f_{1}, f_{2} \in L^{1}\left(\mathbb{R}^{d}\right)$ with compact support we may find $2^{d}$ adjacent congruent cubes $Q_{1}, \ldots Q_{2^{d}} \in \mathcal{D}$, whose union is $\bar{Q}$, such that $\bar{Q}$ contains the support of both $f_{1}, f_{2}$. For each $\ell=1, \ldots, 2^{d}$, referring to the definition (2.1), we inductively set

$$
\mathcal{S}_{\mathcal{D}, \ell, 0}:=\left\{Q_{\ell}\right\}, \quad \mathcal{S}_{\mathcal{D}, \ell, n}:=\bigcup_{Q \in \mathcal{S}_{\mathcal{D}, \ell, n-1}} \mathcal{I}_{Q}, n=1,2, \ldots \quad \mathcal{S}_{\mathcal{D}, \ell}:=\bigcup_{n \geq 1} \mathcal{S}_{\mathcal{D}, \ell, n} .
$$


By using the packing estimate (2.2) and disjointness of $Q_{\ell}$, it is easy to see that

$$
\mathcal{S}_{\mathcal{D}}:=\{\bar{Q}\} \cup \bigcup_{\ell=1}^{2^{d}} \mathcal{S}_{\mathcal{D}, \ell}
$$

is a sparse collection of dyadic cubes. The reason for employing the larger $\bar{Q}$ in place of each $Q_{\ell}$ will be clear below.

2.2. Main line of proof of (1.1). The main thrust of the proof is provided by the lemma below, which we plan to apply iteratively.

Lemma 2.1. Let $f_{1}, f_{2} \in L_{\text {loc }}^{1}\left(\mathbb{R}^{d}\right)$ and $Q \in \mathcal{D}$. For all dyadic shifts $\mathbb{S}$ constructed on $\mathcal{D}$

$$
\left|\mathbb{S}^{\varrho}\left(f_{1} \mathbf{1}_{Q}, f_{2} \mathbf{1}_{Q}\right)\right| \leq C \varrho|Q|\left\langle f_{1}\right\rangle_{Q}\left\langle f_{2}\right\rangle_{Q}+\sum_{I \in \mathcal{I}_{Q}}\left|\mathbb{S}_{\mathcal{D}(I)}^{\varrho}\left(f_{1} \mathbf{1}_{I}, f_{2} \mathbf{1}_{I}\right)\right|
$$

Fixing $f_{1}, f_{2} \in L^{1}\left(\mathbb{R}^{d}\right)$ with compact support and having constructed $\mathcal{S}_{\mathcal{D}}\left(f_{1}, f_{2}\right)$ in the previous subsection, we turn to the proof of (1.1) assuming the lemma. Let $\mathbb{S} e$ be a fixed but arbitrary dyadic shift of complexity $\varrho$ constructed on $\mathcal{D}$. We expand

$$
\mathbb{S}^{\varrho}\left(f_{1}, f_{2}\right)=\mathbb{S}^{\varrho}\left(f_{1} \mathbf{1}_{\bar{Q}}, f_{2} \mathbf{1}_{\bar{Q}}\right)=\sum_{k, \ell=1}^{2^{d}} \mathbb{S}^{\varrho}\left(f_{1} \mathbf{1}_{Q_{k}}, f_{2} \mathbf{1}_{Q_{\ell}}\right)
$$

and estimate the terms $\mathbb{S}^{\varrho}\left(f_{1} \mathbf{1}_{Q_{k}}, f_{2} \mathbf{1}_{Q_{\ell}}\right)$ for $k \neq \ell$. We have for any $R \subset \mathcal{D}$ that

$$
S_{R}\left(f_{1} \mathbf{1}_{Q_{k}}, f_{2} \mathbf{1}_{Q_{\ell}}\right) \begin{cases}=0 & \text { if either } Q_{k} \nsubseteq R \text { or } Q_{\ell} \nsubseteq R \\ =S_{R}\left(\left\langle f_{1}\right\rangle_{Q_{k}} \mathbf{1}_{Q_{k}},\left\langle f_{2}\right\rangle_{Q_{\ell}} 1_{Q_{\ell}}\right) & \text { if } Q_{k}, Q_{\ell} \subset R, \ell(R) \geq 2^{\varrho} \ell\left(Q_{\ell}\right) \\ \text { bounded in abs. value by }\left|Q_{k}\right|\left\langle f_{1}\right\rangle_{Q_{k}}\left\langle f_{2}\right\rangle_{Q_{\ell}} & \text { otherwise. }\end{cases}
$$

The second condition is satisfied since the kernel $s_{R}$ of $S_{R}$ is such that

$$
y \mapsto s_{R}\left(x_{0}, y\right), \quad x \mapsto s_{R}\left(x, y_{0}\right)
$$

are constant on $Q_{k}, Q_{\ell}$ respectively for all $x_{0}, y_{0}$. There are at most $\varrho$ "otherwise" cases. Thus using the $L^{2}$-bound on the second summand in the first right hand side,

$$
\begin{aligned}
& \left|\mathbb{S}^{\varrho}\left(f_{1} \mathbf{1}_{Q_{k}}, f_{2} \mathbf{1}_{Q_{\ell}}\right)\right| \leq \varrho\left|Q_{k}\right|\left\langle f_{1}\right\rangle_{Q_{k}}\left\langle f_{2}\right\rangle_{Q_{\ell}}+\left|\mathbb{S}_{\left\{R: Q_{k}, Q_{\ell} \subset R, \ell(R) \geq 2 \varrho \ell\left(Q_{\ell}\right)\right\}}^{\varrho}\left(\left\langle f_{1}\right\rangle_{Q_{k}} \mathbf{1}_{Q_{k}},\left\langle f_{2}\right\rangle_{Q_{\ell}} \mathbf{1}_{Q_{\ell}}\right)\right| \\
\leq & (\varrho+1)\left|Q_{k}\right|\left\langle f_{1}\right\rangle_{Q_{k}}\left\langle f_{2}\right\rangle_{Q_{\ell}} \leq 2^{d}(\varrho+1)|\bar{Q}|\left\langle f_{1}\right\rangle_{\bar{Q}}\left\langle f_{2}\right\rangle_{\bar{Q}}
\end{aligned}
$$

whence

$$
\sum_{k \neq \ell}\left|\mathbb{S}^{\varrho}\left(f_{1} \mathbf{1}_{Q_{k}}, f_{2} \mathbf{1}_{Q_{\ell}}\right)\right| \leq 2^{2 d}(\varrho+1)|\bar{Q}|\left\langle f_{1}\right\rangle_{\bar{Q}}\left\langle f_{2}\right\rangle_{\bar{Q}} .
$$

We are left with estimating the terms with $k=\ell$ in (2.3). We apply Lemma 2.1 recursively starting from $Q=Q_{k}$. The recursion stops at the $n$-th step, where $n$ is such that $S_{\mathcal{D}(Q)}^{\varrho}=0$ 
for all $Q \in \mathcal{S}_{\mathcal{D}, k, n}$. Such an $n$ exists because of assumption A1. We have

$$
\begin{aligned}
& \left|\mathbb{S}^{\varrho}\left(f_{1} \mathbf{1}_{Q_{k}}, f_{2} \mathbf{1}_{Q_{k}}\right)\right| \leq C \varrho\left|Q_{k}\right|\left\langle f_{1}\right\rangle_{Q_{k}}\left\langle f_{2}\right\rangle_{Q_{k}}+\sum_{Q \in \mathcal{S}_{\mathcal{D}, k, 1}}\left|\mathbb{S}_{\mathcal{D}(Q)}^{\varrho}\left(f_{1} \mathbf{1}_{Q}, f_{2} \mathbf{1}_{Q}\right)\right| \\
\leq & C \varrho\left|Q_{k}\right|\left\langle f_{1}\right\rangle_{Q_{k}}\left\langle f_{2}\right\rangle_{Q_{k}}+C \varrho \sum_{I \in \mathcal{S}_{\mathcal{D}, k, 1}}|I|\left\langle f_{1}\right\rangle_{I}\left\langle f_{2}\right\rangle_{I}+\sum_{Q \in \mathcal{S}_{\mathcal{D}, k, 2}}\left|\mathbb{S}_{\mathcal{D}(Q)}^{\varrho}\left(f_{1} \mathbf{1}_{Q}, f_{2} \mathbf{1}_{Q}\right)\right| \\
\leq & \cdots \leq C \varrho\left|Q_{k}\right|\left\langle f_{1}\right\rangle_{Q_{k}}\left\langle f_{2}\right\rangle_{Q_{k}}+C \varrho \sum_{Q \in \mathcal{S}_{\mathcal{D}, k}}|Q|\left\langle f_{1}\right\rangle_{Q}\left\langle f_{2}\right\rangle_{Q}
\end{aligned}
$$

Summing over $k$, and recalling (2.4), we obtain that (2.3) is bounded by the right hand side of (1.1), with $\mathcal{S}_{\mathcal{D}}$ constructed in the previous subsection, as claimed. Theorem 1 is established, up to the proof of Lemma 2.1.

2.3. Proof of Lemma 2.1. We set

$$
E:=\bigcup_{I \in \mathcal{I}_{Q}} I, \quad \mathcal{G}:=\{R \in \mathcal{D}: R \not \subset E\} .
$$

Then

$$
\mathbb{S}^{\varrho}\left(f_{1} \mathbf{1}_{Q}, f_{2} \mathbf{1}_{Q}\right)=\mathbb{S}_{\mathcal{G}}^{\varrho}\left(f_{1} \mathbf{1}_{Q}, f_{2} \mathbf{1}_{Q}\right)+\sum_{I \in \mathcal{I}_{Q}} \mathbb{S}_{\mathcal{D}(I)}^{\varrho}\left(f_{1} \mathbf{1}_{I}, f_{2} \mathbf{1}_{I}\right)
$$

We further decompose $\mathcal{G}$ into $\varrho$ subcollections $\mathcal{G}^{\prime}$ such that the sidelengths of $R \in \mathcal{G}^{\prime}$ are of the form $2^{\varrho n+m}$ for a fixed $m=0, \ldots, \varrho-1$ and for some integer $n$. Therefore it suffices to prove that

$$
\left|\mathbb{S}_{\mathcal{G}^{\prime}}^{\varrho}\left(f_{1} \mathbf{1}_{Q}, f_{2} \mathbf{1}_{Q}\right)\right| \leq C|Q|\left\langle f_{1}\right\rangle_{Q}\left\langle f_{2}\right\rangle_{Q}
$$

for each of these subcollections $\mathcal{G}^{\prime}$. To do so, we apply the Calderón-Zygmund decomposition to $f_{j} \mathbf{1}_{Q}, j=1,2$, based on the collection of disjoint cubes $I \in \mathcal{I}_{Q}$. By the stopping condition (2.1) and maximality, these cubes have the property that

$$
\left\langle f_{j}\right\rangle_{I} \leq C\left\langle f_{j}\right\rangle_{Q}, \quad j=1,2 .
$$

Therefore, denoting by $f_{j I}$ the average of $f_{j}$ on $I$ and setting

$$
f_{j} \mathbf{1}_{Q}=g_{j}+b_{j}:=\left(f_{j} \mathbf{1}_{E^{c}}+\sum_{I \in \mathcal{I}_{Q}} f_{j I} \mathbf{1}_{I}\right)+\left(\sum_{I \in \mathcal{I}_{Q}} b_{j I}\right), \quad b_{j I}:=\left(f_{j}-f_{j I}\right) \mathbf{1}_{I}
$$

we have for $j=1,2$,

$$
\begin{aligned}
& \left\|g_{j}\right\|_{\infty} \leq C\left\langle f_{j}\right\rangle_{Q}, \\
& \left\|g_{j}\right\|_{2} \leq C|Q|^{\frac{1}{2}}\left\langle f_{j}\right\rangle_{Q}, \\
& \left\|b_{j I}\right\|_{1} \leq C|I|\left\langle f_{j}\right\rangle_{Q}, \quad I \in \mathcal{I}_{Q} .
\end{aligned}
$$

We need to estimate three types of contributions:

$$
\left|\mathbb{S}_{\mathcal{G}^{\prime}}^{\varrho}\left(f_{1} \mathbf{1}_{Q}, f_{2} \mathbf{1}_{Q}\right)\right| \leq\left|\mathbb{S}_{\mathcal{G}^{\prime}}^{\varrho}\left(g_{1}, g_{2}\right)\right|+\left|\mathbb{S}_{\mathcal{G}^{\prime}}^{\varrho}\left(g_{1}, b_{2}\right)\right|+\left|\mathbb{S}_{\mathcal{G}^{\prime}}^{\varrho}\left(b_{1}, g_{2}\right)\right|+\left|\mathbb{S}_{\mathcal{G}^{\prime}}^{\varrho}\left(b_{1}, b_{2}\right)\right|
$$

The $L^{2}$-boundedness and (2.8) yield immediately that

$$
\left|\mathbb{S}_{\mathcal{G}^{\prime}}^{\varrho}\left(g_{1}, g_{2}\right)\right| \leq\left\|g_{1}\right\|_{2}\left\|g_{2}\right\|_{2} \leq C|Q|\left\langle f_{1}\right\rangle_{Q}\left\langle f_{2}\right\rangle_{Q}
$$


The second and third summand in (2.10) are estimated symmetrically. Considering for instance the second summand, we split

$$
\left|\mathbb{S}_{\mathcal{G}^{\prime}}^{\varrho}\left(g_{1}, b_{2}\right)\right| \leq \sum_{I \in \mathcal{I}_{Q}}\left|\mathbb{S}_{\mathcal{G}^{\prime}}^{\varrho}\left(g_{1}, b_{2 I}\right)\right| \leq \sum_{I \in \mathcal{I}_{Q}} \sum_{R \supsetneq I}\left|S_{R}\left(g_{1}, b_{2 I}\right)\right|
$$

Now when $R \supset I$ and $\ell(R) \geq 2^{\varrho} \ell(I)$, the kernel $s_{R}(x, \cdot)$ is constant on $I$ and $b_{2 I}$ has zero average, whence $S_{R}\left(g_{1}, b_{2 I}\right)=0$. Thus $S_{R}\left(g_{1}, b_{2 I}\right)=0$ unless $R=R(I)$, the unique $R \supsetneq I$ with $\ell(R)<2^{\varrho} \ell(I)$. In this case, we estimate, using the normalization of $s_{R}$, (2.7), (2.9)

$$
\left|S_{R}\left(g_{1}, b_{2 I}\right)\right| \leq\left\|g_{1}\right\|_{\infty}\left\|b_{2 I}\right\|_{1} \leq C|I|\left\langle f_{1}\right\rangle_{Q}\left\langle f_{2}\right\rangle_{Q}
$$

Now, summing over $I$ in (2.12) yields that the second summand in (2.10) is also bounded by $C|Q|\left\langle f_{1}\right\rangle_{Q}\left\langle f_{2}\right\rangle_{Q}$. We are left with estimating the fourth summand in (2.10). Let $I_{1}, I_{2} \in \mathcal{I}_{Q}$. Then, reasoning as previously done for $S_{R}\left(g_{1}, b_{2 I}\right)=0$, we notice that $S_{R}\left(b_{1 I_{1}}, b_{2 I_{2}}\right)=0$ unless $R=R\left(I_{1}\right)=R\left(I_{2}\right)$. Preliminarily observe that the intervals $\left\{I \in \mathcal{I}_{Q}: R(I)=R\right\}$ are pairwise disjoint and contained in $R$, so that

$$
\sum_{I: R(I)=R}\left\|b_{j I}\right\|_{1} \leq 2 \sum_{I: R\left(I_{1}\right)=R}\left\|f_{j} \mathbf{1}_{I}\right\|_{1} \leq 2|R|\left\langle f_{j}\right\rangle_{R} \leq C|R|\left\langle f_{j}\right\rangle_{Q}
$$

where the last inequality follows from $R \not \subset E$. Therefore

$$
\begin{aligned}
& \quad\left|\mathbb{S}_{\mathcal{G}^{\prime}}^{\varrho}\left(b_{1}, b_{2}\right)\right| \leq \sum_{R \in \mathcal{G}^{\prime}}\left|S_{R}\left(b_{1}, b_{2}\right)\right| \leq \sum_{R \in \mathcal{G}^{\prime}} \sum_{I_{2}: R\left(I_{2}\right)=R}\left|S_{R}\left(\sum_{I_{1}: R\left(I_{1}\right)=R} b_{1 I_{1}}, b_{2 I_{2}}\right)\right| \\
& \leq \sum_{R \in \mathcal{G}^{\prime}} \sum_{I_{2}: R\left(I_{2}\right)=R}\left\|b_{2 I_{2}}\right\|_{1} \frac{1}{|R|} \sum_{I_{1}: R\left(I_{1}\right)=R}\left\|b_{1 I_{1}}\right\|_{1} \leq C\left\langle f_{1}\right\rangle_{Q} \sum_{R \in \mathcal{G}^{\prime} I_{2}: R\left(I_{2}\right)=R} \sum_{\leq}\left\|b_{2 I_{2}}\right\|_{1} \\
& \leq C\left\langle f_{1}\right\rangle_{Q} \sum_{I_{2} \in \mathcal{I}_{Q}}\left\|f_{2} 1_{I_{2}}\right\|_{1} \leq C|Q|\left\langle f_{1}\right\rangle_{Q}\left\langle f_{2}\right\rangle_{Q},
\end{aligned}
$$

and the fourth summand in (2.10) is also estimated. We have used (2.13) to get the second inequality in the second line of the above display, and the fact that $\left\{I_{2}: R\left(I_{2}\right)=R\right\}$ are disjoint collections over $R \in \mathcal{G}^{\prime}$ to pass to the last line. Collecting (2.11), (2.12) and (2.14) proves the bound (2.5) and completes the proof of Lemma 2.1, and in turn of Theorem 1.

\section{The Vector-VAlued case And A Matrix $A_{2}$ Bound}

In this section, we extend Theorem 1 , as well as its corollaries, to the case of functions taking values in a finite-dimensional Euclidean space $\mathbb{F}^{n}$. We restrict ourselves to $\mathbb{F}=\mathbb{R}$ as the $\mathbb{C}^{n}$-valued case can be easily recovered from the $\mathbb{R}^{2 n}$-valued one.

We begin with defining a handy replacement for the local average of a nonnegative scalar valued function. For a cube $Q \subset \mathbb{R}^{d}$, we set $\Phi(Q)=\left\{\varphi: \mathbb{R}^{d} \rightarrow \mathbb{R},\|\varphi\|_{\infty} \leq 1\right.$, supp $\left.\phi \subset Q\right\}$. Then, for $f \in L_{\text {loc }}^{1}\left(\mathbb{R}^{d} ; \mathbb{R}^{n}\right)$, set

$$
\langle f\rangle_{Q}:=\left\{\frac{1}{|Q|} \int f \varphi \mathrm{d} x: \varphi \in \Phi(Q)\right\} \subset \mathbb{R}^{n} .
$$


It is not hard to see that $\langle f\rangle_{Q}$ is a closed ${ }^{1}$ convex symmetric (that is, invariant under reflection through the origin) set. It is also not hard to see that

$$
\sup _{v \in\langle f\rangle_{Q}}|v| \leq\langle|f|\rangle_{Q}
$$

where the right hand side simply stands for the average of the scalar function $|f|$ on $Q$. We have overloaded the notation since the two concepts are essentially the same if the input is scalar. If $K, H$ are closed convex symmetric sets then their Minkowski product

$$
\{k h: k \in K, h \in H\}
$$

is a closed symmetric interval and $K H$ denotes indifferently the above interval or its right endpoint. With the above notation, we obtain the following uniform domination theorem for the bilinear forms ${ }^{2}$

$$
\mathbb{S}^{\varrho} \otimes \operatorname{Id}_{\mathbb{R}^{n}}\left(f_{1}, f_{2}\right)=\sum_{j=1}^{n} \mathbb{S}^{\varrho}\left(f_{1, j}, f_{2, j}\right) .
$$

Theorem 3. There exists a constant $C$ depending only on the dimensions $d, n$ such that the following holds. For every $f_{1}, f_{2} \in L^{1}\left(\mathbb{R}^{d} ; \mathbb{R}^{n}\right)$ with compact support and every dyadic lattice $\mathcal{D}$ there exists a sparse collection $\mathcal{S}_{\mathcal{D}}$ such that

$$
\sup _{\mathbb{S}^{e}}\left|\mathbb{S}^{\varrho} \otimes \operatorname{Id}_{\mathbb{R}^{n}}\left(f_{1}, f_{2}\right)\right| \leq C \varrho \sum_{Q \in \mathcal{S}_{\mathcal{D}}}|Q|\left\langle f_{1}\right\rangle_{Q}\left\langle f_{2}\right\rangle_{Q}
$$

the supremum being taken over all dyadic shifts $\mathbb{S} e$ of complexity $\varrho$ constructed on $\mathcal{D}$.

We now consider the class of singular integral operators $\overline{\mathcal{U}}=\{\mathfrak{R e} T: T \in \mathcal{U}\}$ where the class $\mathcal{U}$ has been defined in Section 1 , and their canonical extensions to $\mathbb{R}^{n}$-valued functions, which we assume defined on the dense subspace $\mathcal{W}^{n} \subset L^{2}\left(\mathbb{R}^{d} ; \mathbb{R}^{n}\right)$. As for the scalar case, Proposition 1.1 applied componentwise allows us to extend the uniform domination principle of Theorem 3 to such family of singular integral operators.

Theorem 4. There exists a constant $C$ depending only on the dimensions $d, n$ such that the following holds. For every $f_{1}, f_{2} \in \mathcal{W}^{n}$ and having compact support there exists a sparse collection $\mathcal{S}$ such that

$$
\sup _{T \in \overline{\mathcal{U}}}\left|\left\langle T \otimes \operatorname{Id}_{\mathbb{R}^{n}}\left(f_{1}\right), f_{2}\right\rangle\right| \leq C \sum_{Q \in \mathcal{S}}|Q|\left\langle f_{1}\right\rangle_{Q}\left\langle f_{2}\right\rangle_{Q} .
$$

Remark 3.1. We were introduced to definition (3.1) in the context of sparse domination by S. Treil, who, jointly with Nazarov, Petermichl and Volberg, announced the following result ([22], with full proof appearing in [21]): for a standard real-valued Calderón-Zygmund kernel operator and for $f$ in a suitable dense subspace of $L^{2}\left(\mathbb{R}^{d} ; \mathbb{R}^{n}\right)$ there exist $2^{d}$ sparse collections $\mathcal{S}_{1}, \ldots, \mathcal{S}_{2^{d}}$ such that

$$
T \otimes \operatorname{Id}_{\mathbb{R}^{n}}(f)(x) \in \sum_{k=1}^{2^{d}} \sum_{Q \in \mathcal{S}_{k}} C\langle f\rangle_{Q} \mathbf{1}_{Q}(x)
$$

\footnotetext{
${ }^{1}$ The unit ball of $L^{\infty}(Q)$ is weak-* compact.

${ }^{2}$ Here $f_{1, j}$ stands for the $j$-th coordinate of the function $f_{1}$.
} 
almost every $x \in \mathbb{R}^{d}$ where the summation symbols stand for Minkowski sum. We are not aware of the details of their proof at the time of writing; however, our result can be suitably interpreted as the dual form of their theorem.

Finally, we detail an application of Theorem 4 to matrix weighted bounds. We say that $W \in L_{\text {loc }}^{1}\left(\mathbb{R}^{d} ; \mathbb{M}_{n, n}(\mathbb{R})\right)$ is a matrix weight if it is positive semidefinite almost everywhere. We say that a matrix weight $W$ belongs to the class $A_{2}$ if

$$
[W]_{A_{2}}:=\sup _{Q}\left\|\langle W\rangle_{Q}^{\frac{1}{2}}\left\langle W^{-1}\right\rangle_{Q}^{\frac{1}{2}}\right\|^{2}<\infty .
$$

The following estimate on the weighted space $L^{2}(W)$, with norm

$$
\|f\|_{L^{2}(W)}^{2}=\int_{\mathbb{R}^{d}}\left|W^{\frac{1}{2}}(x) f(x)\right|^{2} \mathrm{~d} x
$$

is a rather immediate consequence of the domination Theorem 4 and of the matrix Carleson embedding theorem of Treil and Volberg [23]. The derivation from Theorem 4 borrows from the approach of Bickel and Wick [3] to the analogous estimate for $\mathbb{R}^{n}$-valued sparse averaging operators.

Corollary 5. Let $W \in L_{\text {loc }}^{1}\left(\mathbb{R}^{d} ; \mathbb{M}_{n, n}(\mathbb{R})\right)$ be an $A_{2}$ matrix weight. Then there holds

$$
\sup _{T \in \overline{\mathcal{U}}}\left\|T \otimes \operatorname{Id}_{\mathbb{R}^{n}}\right\|_{L^{2}(W) \rightarrow L^{2}(W)} \leq C[W]_{A_{2}}^{\frac{3}{2}}
$$

where the positive constant $C$ depends on the dimensions $n, d$ only.

Remark 3.2. Previous partial results on sharp dependence of the $L^{2}$ weighted operator norms of $T$ on the matrix $A_{2}$ characteristic, as well as related work on matrix two-weight inequalities, can be found in $[1,2,4]$ and references therein. The $3 / 2$ power in our estimate is currently the best known, but, unlike the scalar case, we have no indication of it being sharp. The previously mentioned domination theorem (3.3) announced by Nazarov, Petermichl, Treil and Volberg [22] yields the same 3/2 power; see [21] for details.

After a preliminary convex set-valued Calderón-Zygmund lemma in the upcoming Subsection 3.1, we detail the proof of Theorem 3 in Subsection 3.2 and the derivation of the weighted Corollary 5 in the concluding Subsection 3.3.

3.1. A convex set-valued Calderón-Zygmund lemma. Before the actual proof of Theorem 3, we need an analogue of the Calderón-Zygmund lemma based on the convex sets $\langle f\rangle_{Q}$.

Lemma 3.3. Let $A>n^{2}, f \in L_{\text {loc }}^{1}\left(\mathbb{R}^{d} ; \mathbb{R}^{n}\right)$ and $Q \subset \mathbb{R}^{d}$ be a cube. Then the collection $\mathcal{I}_{Q, f}$ of the maximal dyadic subcubes of $Q$ with

$$
\langle f\rangle_{I} \not \subset A\langle f\rangle_{Q}
$$

has the following properties:

$$
\begin{gathered}
\langle f\rangle_{I} \subset 2^{d} A\langle f\rangle_{Q}, \\
\sum_{I \in \mathcal{I}_{Q, f}}|I|<\frac{n^{2}}{A}|Q| .
\end{gathered}
$$


In the proof, we will use the notion of John ellipsoid. If $K \subset \mathbb{R}^{n}$ is a closed convex symmetric set then $\mathcal{E}_{K}$, the John ellipsoid, is the solid ellipsoid of largest volume contained in $K$. This is a closed set with the property that

$$
\mathcal{E}_{K} \subset K \subset \sqrt{n} \mathcal{E}_{K}
$$

where as usual the above denotes concentric dilation.

Proof of Lemma 3.3. We first prove (3.4) which is rather immediate. It is easy to see that if $\widetilde{I}$ is the dyadic parent of $I$ then $\langle f\rangle_{I} \subset 2^{d}\langle f\rangle_{\widetilde{I}}$ and the latter set is contained in $2^{d} A\langle f\rangle_{Q}$ by maximality of $I$.

We come to the proof of (3.5). Here we notice that the collection $\mathcal{I}_{Q, f}$ is invariant under action of $\mathrm{GL}_{n}(\mathbb{R})$. For this reason, there is no loss in generality with assuming that the John ellipsoid of $\langle f\rangle_{Q}$ is the closed unit ball $B$. We say that $I \in \mathcal{I}_{Q, f}$ is of type $j, j=1, \ldots, n$ if there exists $F_{I} \in\langle f\rangle_{I}$ with $\sqrt{n}\left(F_{I}\right)_{j}>A$ : here and below $\left(F_{I}\right)_{j}$ is the $j$-th coordinate. Since $\langle f\rangle_{I} \not \subset A\langle f\rangle_{Q}$, and a fortiori $\langle f\rangle_{I} \not \subset A B$, it follows that each $I$ is of type $j$ for at least one $j=1, \ldots, n$. Let $\mathcal{I}_{j}$ be those $I \in \mathcal{I}_{Q, f}$ of type $j$. We will prove that

$$
\sum_{I \in \mathcal{I}_{j}}|I|<\frac{n}{A}|Q|
$$

which in light of the previous observations yields (3.5). We may find $\varphi_{I} \in \Phi(I)$ such that

$$
F_{I}=\frac{1}{|I|} \int f \varphi_{I} \mathrm{~d} x \text {. }
$$

Define now

$$
F_{Q}:=\sum_{I \in \mathcal{I}_{j}} \frac{|I|}{|Q|} F_{I}=\frac{1}{|Q|} \int f \varphi_{Q}, \quad \varphi_{Q}:=\sum_{I \in \mathcal{I}_{j}} \varphi_{I} .
$$

Since $I$ are pairwise disjoint and contained in $Q, \varphi_{Q} \in \Phi(Q)$. This means that $F_{Q} \in\langle f\rangle_{Q} \subset$ $\sqrt{n} B$. In particular $\left(F_{Q}\right)_{j} \leq \sqrt{n}$. But then, applying the type $j$ condition in the last step

$$
\sqrt{n} \geq\left(F_{Q}\right)_{j}=\sum_{I \in \mathcal{I}_{j}} \frac{|I|}{|Q|}\left(F_{I}\right)_{j}>\frac{A}{\sqrt{n}} \sum_{I \in \mathcal{I}_{j}} \frac{|I|}{|Q|}
$$

which is (3.6). The proof is thus complete.

3.2. Proof of Theorem 3. The proof of the vector-valued version follows the exact same outline of the proof of Theorem 1. We just detail the main iterative step, which is carried out through a vector-valued version of Lemma 2.1.

Lemma 3.4. Let $f_{1}, f_{2} \in L_{\text {loc }}^{1}\left(\mathbb{R}^{d} ; \mathbb{R}^{n}\right)$ and $Q \in \mathcal{D}$. Let $\mathcal{I}_{Q}$ be the collection of maximal elements of $\mathcal{I}_{Q, f_{1}} \cup \mathcal{I}_{Q, f_{2}}$. Then, for all dyadic shifts $\mathbb{S}$ constructed on $\mathcal{D}$

$$
\left|S^{\varrho} \otimes \operatorname{Id}_{\mathbb{R}^{n}}\left(f_{1} \mathbf{1}_{Q}, f_{2} \mathbf{1}_{Q}\right)\right| \leq C \varrho|Q|\left\langle f_{1}\right\rangle_{Q}\left\langle f_{2}\right\rangle_{Q}+\sum_{I \in \mathcal{I}_{Q}}\left|\mathbb{S}_{\mathcal{D}(I)}^{\varrho} \otimes \operatorname{Id}_{\mathbb{R}^{n}}\left(f_{1} \mathbf{1}_{I}, f_{2} \mathbf{1}_{I}\right)\right|
$$

where $C$ is a positive absolute constant depending on the dimensions $n, d$ only. 
We clarify that, in the context of functions $f_{1}, f_{2} \in L_{\mathrm{loc}}^{1}\left(\mathbb{R}^{d} ; \mathbb{R}^{n}\right)$, the collection $\mathcal{I}_{Q, f_{j}}$ refers to the one defined in Lemma 3.3 for the value $A=2^{8} n^{2}$. Thus $\mathcal{I}_{Q}$ defined in Lemma 3.4 above satisfy (2.2) just like in the scalar case.

Proof of Lemma 3.4. In this proof, the constant $C$ is meant to depend on $n, d$ only and may vary between instances.

We limit ourselves to indicating the necessary changes from the argument for Lemma 2.1. We start by setting

$$
E:=\bigcup_{I \in \mathcal{I}_{Q}} I, \quad \mathcal{G}:=\{R \in \mathcal{D}: R \not \subset E\} .
$$

Then similarly to the scalar case,

$$
\mathbb{S}^{\varrho} \otimes \operatorname{Id}_{\mathbb{R}^{n}}\left(f_{1} \mathbf{1}_{Q}, f_{2} \mathbf{1}_{Q}\right)=\mathbb{S}_{\mathcal{G}}^{\varrho} \otimes \operatorname{Id}_{\mathbb{R}^{n}}\left(f_{1} \mathbf{1}_{Q}, f_{2} \mathbf{1}_{Q}\right)+\sum_{I \in \mathcal{I}_{Q}} \mathbb{S}_{\mathcal{D}(I)}^{\varrho} \otimes \operatorname{Id}_{\mathbb{R}^{n}}\left(f_{1} \mathbf{1}_{I}, f_{2} \mathbf{1}_{I}\right)
$$

It thus suffices to prove that

$$
\left|\mathbb{S}_{\mathcal{G}}^{\varrho} \otimes \operatorname{Id}_{\mathbb{R}^{n}}\left(f_{1} \mathbf{1}_{Q}, f_{2} \mathbf{1}_{Q}\right)\right| \leq C \varrho|Q|\left\langle f_{1}\right\rangle_{Q}\left\langle f_{2}\right\rangle_{Q}
$$

To prove (3.7), it is useful to transform the John ellipsoids of $\left\langle f_{1}\right\rangle_{Q},\left\langle f_{2}\right\rangle_{Q}$ to the closed unit ball $B$, which can be achieved via actions of $\mathrm{GL}_{n}(\mathbb{R})$. For fixed $f_{1}, f_{2}$, there exists matrices $A_{1}, A_{2} \in \mathrm{GL}_{n}(\mathbb{R})$ such that for $j=1,2, A_{j} \tilde{f}_{j}=f_{j}$ and the John ellipsoid of $\left\langle\tilde{f}_{j}\right\rangle_{Q}$ is $B$. We claim that

$$
\left|\mathbb{S}_{\mathcal{G}}^{\varrho}\left(\tilde{f}_{1, k_{1}} \mathbf{1}_{Q}, \tilde{f}_{2, k_{2}} \mathbf{1}_{Q}\right)\right| \leq C \varrho|Q|, \quad \forall k_{1}, k_{2}=1, \ldots, n .
$$

Assuming (3.8), let us first explain how it implies (3.7) and thus the result of the lemma. A simple calculation shows that

$$
\begin{aligned}
& \left|\mathbb{S}_{\mathcal{G}}^{\varrho} \otimes \operatorname{Id}_{\mathbb{R}^{n}}\left(f_{1} \mathbf{1}_{Q}, f_{2} \mathbf{1}_{Q}\right)\right|=\left|\mathbb{S}_{\mathcal{G}}^{\varrho} \otimes \operatorname{Id}_{\mathbb{R}^{n}}\left(\left(A_{1} \tilde{f}_{1}\right) \mathbf{1}_{Q},\left(A_{2} \tilde{f}_{2}\right) \mathbf{1}_{Q}\right)\right| \\
= & \left|\sum_{j, k_{1}, k_{2}=1}^{n} A_{1}^{j k_{1}} A_{2}^{j k_{2}} \mathbb{S}_{\mathcal{G}}^{\varrho}\left(\tilde{f}_{1, k_{1}} \mathbf{1}_{Q}, \tilde{f}_{2, k_{2}} \mathbf{1}_{Q}\right)\right| \leq C \varrho|Q| \sup _{1 \leq k_{1}, k_{2} \leq n}\left|\sum_{j=1}^{n} A_{1}^{j k_{1}} A_{2}^{j k_{2}}\right| .
\end{aligned}
$$

We have used (3.8) in the last step. Then (3.7) will follow if we show that

$$
\left|\sum_{j=1}^{n} A_{1}^{j k_{1}} A_{2}^{j k_{2}}\right|=\left|\left(A_{1} e_{k_{1}}\right)\left(A_{2} e_{k_{2}}\right)\right| \leq\left\langle f_{1}\right\rangle_{Q}\left\langle f_{2}\right\rangle_{Q} \quad \forall 1 \leq k_{1}, k_{2} \leq n
$$

where $e_{k}$ is the $k$-th coordinate vector. Fix $1 \leq k_{1}, k_{2} \leq n$. By virtue of $e_{k_{j}} \in B \subset\left\langle\tilde{f}_{j}\right\rangle_{Q}$, there exists $\varphi_{j} \in \Phi(Q)$ such that

$$
e_{k_{j}}=\frac{1}{|Q|} \int \tilde{f}_{j} \varphi_{j}, \quad j=1,2
$$

Therefore,

$$
A_{j} e_{k_{j}}=\frac{1}{|Q|} \int\left(A_{j} \tilde{f}_{j}\right) \varphi_{j}=\frac{1}{|Q|} \int f_{j} \varphi_{j} \in\left\langle f_{j}\right\rangle_{Q},
$$

which implies immediately the claimed (3.9) and hence (3.7). 
Now we turn to the proof of (3.8). We operate the same Calderón-Zygmund decomposition of $\tilde{f}_{1, k_{j}} \mathbf{1}_{Q} j=1,2$ as (2.6), this time with respect to the cubes $\mathcal{I}_{Q}$ defined in this context. The analogues of (2.7) and (2.9) are, for $j=1,2$

$$
\begin{array}{ll}
\tilde{g}_{j}(x) \in C\left\langle\tilde{f}_{j}\right\rangle_{Q} \subset C B & \forall x \in Q, \\
\left\langle\tilde{b}_{j I}\right\rangle_{I} \subset C\left\langle\tilde{f}_{j}\right\rangle_{Q} \subset C B & \forall I \in \mathcal{I}_{Q} .
\end{array}
$$

An immediate consequence of (3.10), (3.11) is that each coordinate of $\tilde{g}_{j}, \tilde{b}_{j}$ satisfies scalar estimates analogous to (2.8) and (2.9): for $j=1,2$ and $1 \leq k_{j} \leq n$

$$
\left\|\tilde{g}_{j, k_{j}}\right\|_{2} \leq C|Q|^{\frac{1}{2}}, \quad\left\|\tilde{b}_{j I, k_{j}}\right\|_{1} \leq C|I| .
$$

By virtue of these estimates and of the fact that the average of each coordinate of $\tilde{b}_{j I}$ vanishes on $I$, the estimate (3.8) follows by repeating the proof of (2.10) from the scalar case.

3.3. Proof of Corollary 5. Let $T \in \overline{\mathcal{U}}$ and $W$ be a $n \times n$ matrix $A_{2}$ weight on $\mathbb{R}^{d}$. For convenience we write $V_{1}=W^{-1}, V_{2}=W$. Since

$$
\left\|T \otimes \operatorname{Id}_{\mathbb{R}^{n}}\right\|_{L^{2}(W) \rightarrow L^{2}(W)}=\sup \left\{\left|\left\langle T \otimes \operatorname{Id}_{\mathbb{R}^{n}}\left(V_{1} f_{1}\right), V_{2} f_{2}\right\rangle\right|:\left\|f_{1}\right\|_{L^{2}\left(V_{1}\right)}=\left\|f_{2}\right\|_{L^{2}\left(V_{2}\right)}=1\right\}
$$

by virtue of the domination Theorem 4 it suffices to show that whenever $\mathcal{S}$ is a sparse collection and $\left\|f_{1}\right\|_{L^{2}\left(V_{1}\right)}=\left\|f_{2}\right\|_{L^{2}\left(V_{2}\right)}=1$ there holds

$$
\sum_{Q \in \mathcal{S}}|Q|\left\langle V_{1} f_{1}\right\rangle_{Q}\left\langle V_{2} f_{2}\right\rangle_{Q} \leq C[W]_{A_{2}}^{\frac{3}{2}}
$$

Fix such a collection $\mathcal{S}$ and $f_{1}, f_{2}$. By definition of $\langle\cdot\rangle_{Q}$, for each $Q$ we may find $\phi_{j Q} \in \Phi(Q)$ such that

$$
\left\langle V_{1} f_{1}\right\rangle_{Q}\left\langle V_{2} f_{2}\right\rangle_{Q}=F_{1 Q} F_{2 Q}, \quad F_{j Q}:=\frac{1}{|Q|} \int V_{j} f_{j} \phi_{j Q} \mathrm{~d} x, \quad j=1,2 .
$$

A similar reduction to the one carried out in [3, Proof of Theorem 1.4] then yields that

$$
\sum_{Q \in \mathcal{S}}|Q|\left\langle V_{1} f_{1}\right\rangle_{Q}\left\langle V_{2} f_{2}\right\rangle_{Q} \leq[W]_{A_{2}}^{\frac{1}{2}} \prod_{j=1,2}\left(\sum_{Q \in \mathcal{S}}|Q|\left|\left\langle V_{j}\right\rangle_{Q}^{-\frac{1}{2}} F_{j Q}\right|^{2}\right)^{\frac{1}{2}}
$$

which de facto reduces (3.12) to proving that, for $j=1,2$

$$
\left.\sum_{Q \in \mathcal{S}}|Q|\left\langle V_{j}\right\rangle_{Q}^{-\frac{1}{2}} F_{j Q}\right|^{2}=\sum_{Q}\left(A_{j Q} F_{j Q}\right) F_{j Q} \leq C\left[V_{j}\right]_{A_{2}}
$$

where we set

$$
A_{j Q}= \begin{cases}0 & Q \notin \mathcal{S} \\ |Q|\left\langle V_{j}\right\rangle_{Q}^{-1} & Q \in \mathcal{S}\end{cases}
$$


and recall that $\left[V_{j}\right]_{A_{2}}=[W]_{A_{2}}$. Note that the matrices $A_{j Q}$ satisfy the following packing condition: for any $R \in \mathcal{S}$,

$$
\frac{1}{|R|} \sum_{Q \subset R}\left\|\left\langle V_{j}\right\rangle_{Q}^{\frac{1}{2}} A_{j Q}\left\langle V_{j}\right\rangle_{Q}^{\frac{1}{2}}\right\|=\frac{1}{|R|} \sum_{\substack{Q \subset R \\ Q \in \mathcal{S}}}|Q|\left\|\left\langle V_{j}\right\rangle_{Q}^{\frac{1}{2}}\left\langle V_{j}\right\rangle_{Q}^{-1}\left\langle V_{j}\right\rangle_{Q}^{\frac{1}{2}}\right\|=\frac{1}{|R|} \sum_{\substack{Q \subset R \\ Q \in \mathcal{S}}}|Q| \leq C
$$

by virtue of the fact that every sparse collection is Carleson: see [17]. Estimate (3.15) is then a consequence of the Carleson embedding theorem initially due to Treil and Volberg [23], in the form recalled in [3, Theorem 1.3]. The linear behavior of the constant in [3, Theorem 1.3] has been first obtained by Isralowitz, Kwon and Pott in [13, Theorem 1.3]. We remark that, while in [13, Theorem 1.3] $F_{j Q}$ defined in (3.13) corresponds to the precise choice $\phi_{j Q}=1_{Q}$, the proof works just as well for any choice $\phi_{j Q} \in \Phi(Q)$. This completes the proof of (3.15) and in turn, of Corollary 5.

\section{REFERENCES}

[1] Kelly Bickel, Amalia V. Culiuc, Sergei Treil, and Brett D. Wick, Two weight estimates with matrix measures for well localized operators, under completion.

[2] Kelly Bickel, Stefanie Petermichl, and Brett D. Wick, Bounds for the Hilbert transform with matrix $A_{2}$ weights, J. Funct. Anal. 270 (2016), no. 5, 1719-1743. MR 3452715

[3] Kelly Bickel and Brett D. Wick, A study of the matrix Carleson embedding theorem with applications to sparse operators, J. Math. Anal. Appl. 435 (2016), no. 1, 229-243. MR 3423392

[4] _ Well-localized operators on matrix weighted $L^{2}$ spaces, Houston J. Math. 42 (2016), no. 1, 249283. MR 3502781

[5] José M. Conde-Alonso and Xavier Parcet, Nondoubling Calderón-Zygmund theory -a dyadic approach, preprint arXiv:1604.03711.

[6] José M. Conde-Alonso and Guillermo Rey, A pointwise estimate for positive dyadic shifts and some applications, Math. Ann. 365 (2016), no. 3-4, 1111-1135. MR 3521084

[7] Amalia Culiuc, Francesco Di Plinio, and Yumeng Ou, Domination of multilinear singular integrals by positive sparse forms, preprint arXiv:1603.05317.

[8] Francesco Di Plinio and Yumeng Ou, A modulation invariant Carleson embedding theorem outside local $L^{2}$, preprint arXiv:1506.05827, to appear in J. Anal. Math.

[9] Ana Grau de la Herrán and Tuomas Hytönen, Dyadic representation and boundedness of non-homogeneous calderón-zygmund operators with mild kernel regularity, preprint arXiv: 1612.05133.

[10] Tuomas P. Hytönen, Representation of singular integrals by dyadic operators, and the $A_{2}$ theorem, preprint arXiv:1108.5119.

[11] _ The sharp weighted bound for general Calderón-Zygmund operators, Ann. of Math. (2) 175 (2012), no. 3, 1473-1506. MR 2912709

[12] The $A_{2}$ theorem: remarks and complements, Harmonic analysis and partial differential equations, Contemp. Math., vol. 612, Amer. Math. Soc., Providence, RI, 2014, pp. 91-106. MR 3204859

[13] Joshua Isralowitz, Hyun Kyoung Kwon, and Sandra Pott, A matrix weighted T1 theorem for matrix kernelled Calderón-Zygmund operators - $i$, preprint arXiv:1401.6570.

[14] Michael Lacey, An elementary proof of the $A_{2}$ Bound, preprint arXiv:1501.05818.

[15] Michael T. Lacey and Dario Mena Arias, The sparse T1 Theorem, preprint arXiv:1610.01531, to appear in Houston Math. J.

[16] Andrei Lerner, On pointwise estimates involving sparse operators, preprint arXiv:1512.07247 (2015).

[17] Andrei Lerner and Fedor Nazarov, Intuitive dyadic calculus: the basics, preprint arXiv:1508.05639 (2015).

[18] Andrei K. Lerner, A simple proof of the $A_{2}$ conjecture, Int. Math. Res. Not. IMRN (2013), no. 14, 31593170. MR 3085756 
[19] Kangwei Li, Kabe Moen, and Wenchang Sun, The sharp weighted bound for multilinear maximal functions and Calderón-Zygmund operators, J. Fourier Anal. Appl. 20 (2014), no. 4, 751-765. MR 3232584

[20] F. Nazarov, S. Treil, and A. Volberg, The T b-theorem on non-homogeneous spaces, Acta Math. 190 (2003), no. 2, 151-239. MR 1998349

[21] Fedor Nazarov, Stefanie Petermichl, Serger Treil, and Alexander Volberg, Convex body domination and weighted estimates with matrix weights, preprint arXiv:1701.01907.

[22] Sergei Treil, personal communication, Spring 2016.

[23] Sergei Treil and Alexander Volberg, Wavelets and the angle between past and future, J. Funct. Anal. 143 (1997), no. 2, 269-308. MR 1428818

School of Mathematics, Georgia Institute of Technology, Atlanta, GA 30332, USA (A. Culiuc)

Department of Mathematics, University of Virginia, Kerchof Hall, Box 400137, Charlottesville, VA 22904-4137, USA (F. Di Plinio)

Department of Mathematics, Massachusetts Institute of Technology, 77 Massachusetts Avenue, Cambridge, MA 02139, USA (Y. Ou)

E-mail address, A. Culiuc: amalia@math.gatech.edu

E-mail address, F. Di Plinio: francesco.diplinio@virginia.edu

E-mail address, Y. Ou: yumengou@mit.edu 